\title{
An unusual presentation of a metastatic adenocarcinoma of the cervix with a rectovaginal fistula arising from a primary sigmoid adenocarcinoma in the postpartum period - case report
}

\author{
Harjit S Dhaliwal, Rachana Dwivedi, Tharanga Gunasekera, Jasmina Glogic
}

\author{
Corresponding author: Dr. Harjit S Dhaliwal, Department of Obstetrics and Gynecology and \\ Histopathology, Royal Bournemouth Hospital, Bournemouth, UK; \\ Email : harjitdhaliwal2004@yahoo.co.uk
}

Distributed under Attribution-Non Commercial - Share Alike 4.0 International (CC BY-NC-SA 4.0)

\begin{abstract}
Colorectal cancer in pregnancy and in the postpartum period is rare. We report a case of a 38-year-old woman with metastatic adenocarcinoma of the cervix in the postpartum period. Due to the rarity and lack of insight of this condition, she visited various clinicians and was finally referred to secondary care based on feculent material seen vaginally and also palpation of an abdominal mass in the left iliac fossa. Cervical biopsy confirmed a colorectal primary and CT and MRI, which showed a locally advanced tumor invading the posterior cervical and anterior rectal wall. MRI suggested T4N2. Given the locally advanced stage, the patient was counseled by the colorectal surgeons the need for neoadjuvant therapy with chemo or radiotherapy prior to embarking on surgery with the possibility of adjuvant therapy thereafter. Colorectal cancer in pregnancy and the postpartum period is associated with challenges being clinical, diagnostic and therapeutic which often leads to a delayed diagnosis in the advanced stage, which invariably carries a poor prognosis.
\end{abstract}

Keywords: Uterine cervix, sigmoid cancer, pregnancy.

Colorectal cancer (CRC) in pregnancy and in the postpartum period represents as a distinct entity compared to the general population with limited management guidance available to the clinician. Due to the paucity in the literature with fewer than 300 reported cases, the diagnosis is often significantly delayed as there is an overlap of signs and symptoms between these tumors and the physiological adaptations in pregnancy. Colorectal rectal cancers presenting during pregnancy and in the postpartum period is a rare occurrence with an incidence of $0.002-0.2 \%{ }^{1}$ In young patients, below the age of 40 it is occasionally associated with genetic syndromes (Lynch, Familial adenomatous polyposis, Gardner, Peutz-Jeghers and familial colorectal), and longstanding inflammatory bowel disease (Chrons,
Ulcerative colitis).

Early diagnosis is one of the most important prognostic factors in CRC. Stage for stage survival was worse when compared to non- pregnant women. The prognosis with a 5year survival rate ranges between $0-42$ percent in comparison to the general population ${ }^{2}$. The reason for a delayed diagnosis could be the due to overlap of common symptoms of pregnancy with that of CRC- nausea, vomiting, abdominal pains, anemia and rectal bleeding. In a study of 119 patients with CRC in pregnancy, Pellino et al reported a high incidence of rectal bleeding $47 \%$, followed by abdominal pains in $37.6 \%$ and constipation in $14 \%$, obstruction in $9.4 \%$ and perforation in $2.4 \%$ in this cohort of women ${ }^{3}$. Hence, rectal bleeding during pregnancy should warrant further

Received: $6^{\text {th }}$ March 2020, Peer review completed: $19^{\text {th }}$ August 2020, Accepted: $1^{\text {th }}$ October 2020.

Dhaliwal HS, Dwivedi R, Gunasekera T, Glogic J. An unusual presentation of a metastatic adenocarcinoma of the cervix with a rectovaginal fistula arising from a primary sigmoid adenocarcinoma in the postpartum period - case report. The New Indian Journal of OBGYN. 2021; 8(1): 139-42. 
assessment as one need to exclude other differentials such as hemorrhoids, IBD (inflammatory bowel disease), infectious colitis and CRC.

\section{Case}

A 38-year-old woman presented to our department with intermittent diarrhea since the delivery of her child by caesarean section. She had no significant medical or a surgical history. She was a low risk multigravida. Booking weight was 65 kilos. She had a couple of growth scans in the antenatal period. They showed a normally grown fetus with normal doppler's and liquor volume. She went into spontaneous labour at 41 weeks of gestation and had an emergency caesarean section for fetal distress. It was an uncomplicated surgical procedure. She was discharged on day 2 with an uneventful postnatal check. On day three the patient felt generally unwell. Clinical examination and observations were unremarkable. She did not have any abdominal pain or an offensive vaginal discharge. The surgical scar was healing well. The only new symptom she had was diarrhea. There was no loss of appetite. Over the course of the day, she felt much better and was reassured.

She then presented to her general practitioner eight weeks later with a two-week history of continuous diarrhea. It was worse after eating. There was fecal urgency with episodes of incontinence. She felt that her appetite was poor and had lost weight. She also had developed abdominal cramps. Examination suggested she was tender on the left side of her abdomen. Bowel sounds were normal. Her recorded weight was $55.7 \mathrm{~kg}$. A loss of approximately 10 kilos from her booking weight. Stools samples were sent for a presumed diagnosis of an infected diarrhea (Giardiasis/ Campylobacter). She was given dioralyte sachets. The following day a different general practitioner reviewed her. The diarrhea continued to persist and the patient had now noticed some fecal matter in the vagina. Examination revealed tenderness in the left iliac fossa but no mass was felt. She was advised that it was unlikely to be a fistula related to her caesarean delivery. The patient had a follow up a week later, with her same doctor. The diarrhea was settling. Observations were unremarkable and the stool samples were negative for any parasite, ova and cysts. Her blood test suggested an iron deficient picture. She was commenced on oral iron supplements.

A week later she presented herself to a different general practitioner based in the same surgery with a two days history of feeling unwell with fever, pelvic pain and a brownish vaginal discharge. Her weight loss and decreased appetite continued. Her recorded weight at presentation was 53 kilos. She was then referred to ourselves.

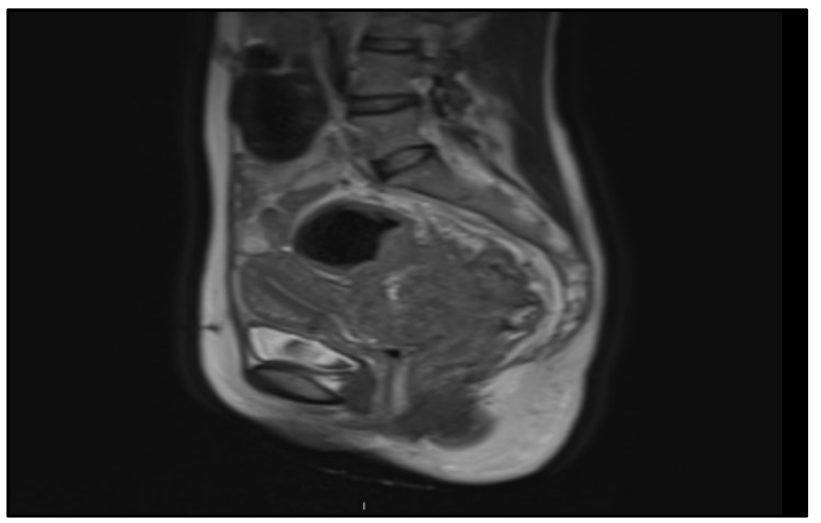

Figure 1: Cervical invasion by sigmoid tumor

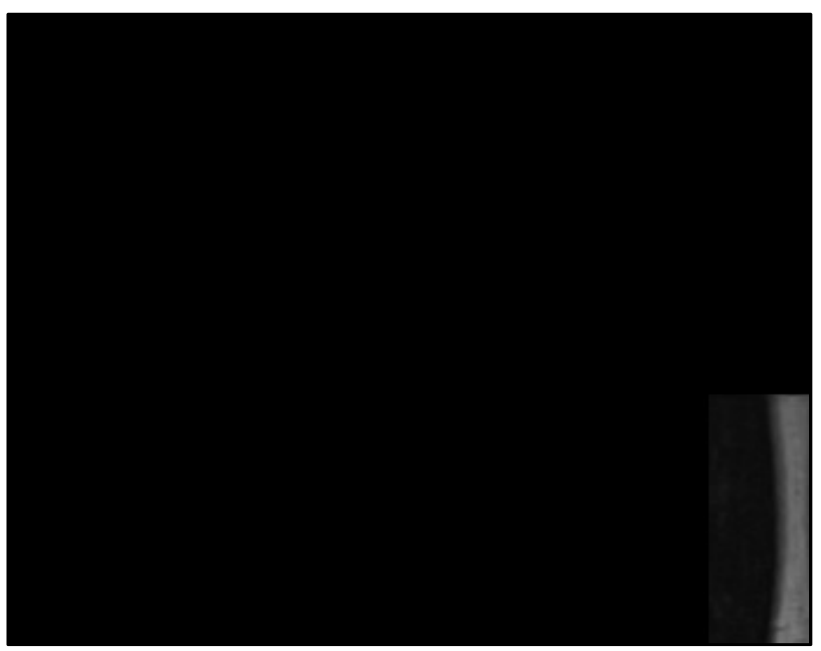

Figure 2: Rectovaginal fistula

Clinical examination suggested an abdominal mass in the left iliac fossa and a large fungating cervical mass on vaginal examination. Feculent material was also seen on speculum examination. A computed tomogram (CT) scan suggested a very large necrotic cervical tumour that was filling the lower pelvis and also invading the adjacent rectum. Normal appearances of the liver, spleen, and kidneys adrenal were documented on the CT findings. An magnetic resonance imaging (MRI) suggested that there was a large sigmoid tumour that was invading the posterior cervical and anterior rectal wall (figure - 1,2). There were also numerous enlarged lymph nodes (presacral, meso-rectal, right common iliac and a suspicious left external iliac node). MRI staging suggested T4N2 in accordance with the TMN classification. CT chest suggested no evidence of any mediastinal lymphadenopathy or destructive bone lesions. 


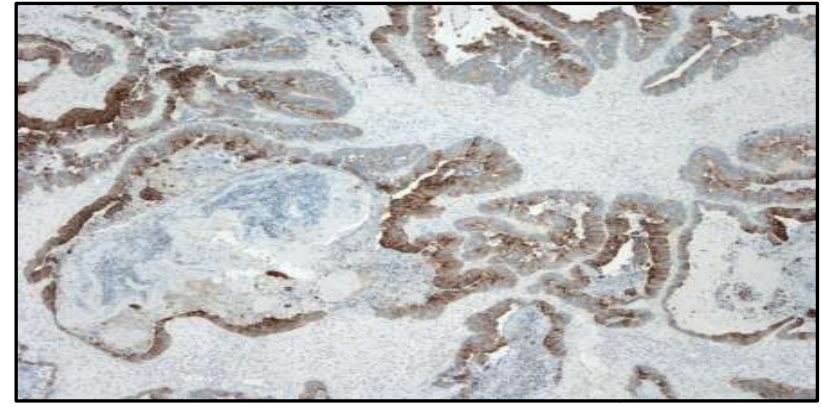

Figure 3: Strong nuclear staining by CK20 marker

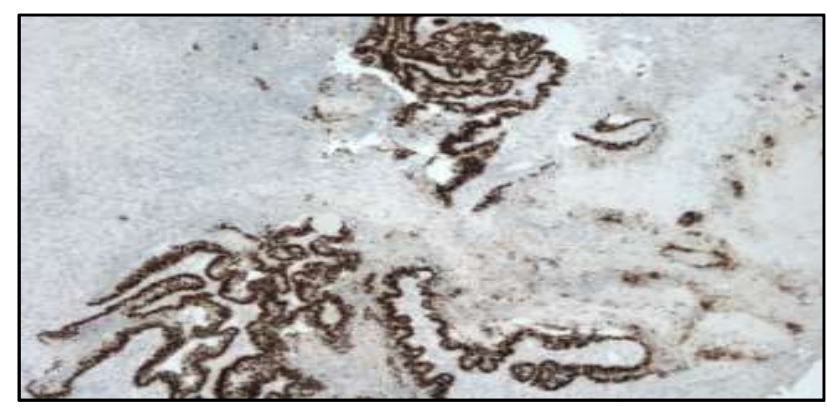

Figure 4: Strong nuclear staining of tumour cells by the CDX2 marker

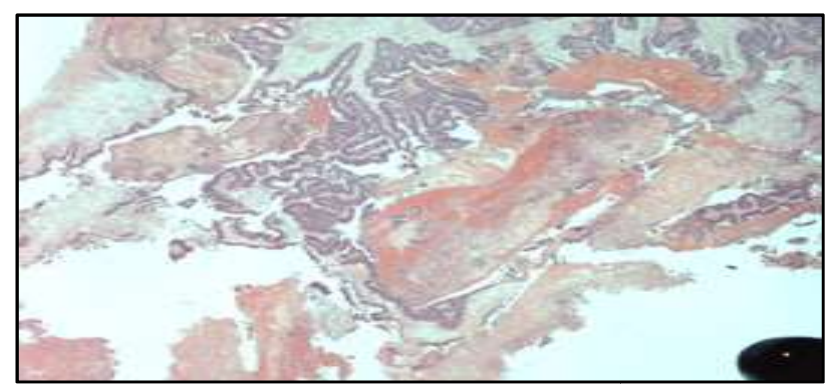

Figure 5: Cervical biopsy showing metastatic moderately differentiated adenocarcinoma cells

A cervical biopsy was performed. It suggested a moderately differentiated endometriod type adenocarcinoma. Immunohistochemistry suggested that the tumour cells had a strong positivity for CEA and CK20 and CDX2 (figure 3, 4). The tumour cells were negative for the ER, Vimentin and CK7 stains. The immunoprofile was in favour of a colorectal origin metastatic adenocarcinoma (figure 5).

Genetic testing on the tumour sample for BRAF, KRAS, NRAS, TP53, MMR and PIK3CA suggested no evidence of any detectable mutations. KRAS and NRAS gene mutation analysis revealed wild type colonic adenocarcinoma without mutation in codons 12,13 and 61and 146. The comments from the histopathologist were that, the patient may respond to epidermal growth factor receptor (EGFR) monoclonal antibody therapies.
Given the locally advanced stage and the involvement of several lymph nodes, the patient was counseled for the need for neo adjuvant chemotherapy for a 6 weeks period before any surgical intervention with the possibility of long course of chemo radiotherapy as well.

Due to the constrains of having two young children with no family based in the UK, and such intense treatment would have been be very difficult to cope with, without a significantly better family support. The couple eventually decided to relocate to their country of origin and pursue cancer treatment options there. She was counseled; that operating too early without pre-treatment with chemo or radiotherapy and by an inexperienced surgeon would leave residual tumor in the pelvis that will recur rapidly and aggressively.

\section{Discussion}

The pathogenesis of CRC in pregnancy and in the postpartum period is unclear and poorly understood. Initial studies had evaluated the role of pregnancy related oestrogen and progesterone as a cause for CRC in pregnancy and in the postpartum ${ }^{4,5}$. This together with other stimulating growth hormones lead to an increase in cancer cell division and induction of CRC. None of these studies included patients with $\mathrm{CRC}$ in the postpartum period. A study by J Silverstein et al, postpartum colorectal cancer ( 5 cases), found a lack of detectable ER and PR expressions and concluded it was an unlikely cause for the accelerated pathogenesis of CRC ${ }^{6}$. The cancer genome atlas RNA sequencing data for CRC further supported this ${ }^{7}$. There was also a lack of ER expression in our patient. It could be due to the downregulation of the hormones and receptors in the postpartum period.

Various other theories have been proposed that lead to the aggressive nature of $\mathrm{CRC}$ in pregnancy and in the postpartum period. These include the abundance of cyclooxygenase-2, insulin-like growth factor family (IGF$1, \mathrm{II})$ and pro-angiogenic factors such as placental growth factor and soluble fms-like tyrosine kinase (sFlt-1). Finally, the immunosuppressive state of pregnancy could also be a contributor in lowering the host defenses against an aggressive production of malignant cells.

Metastasis to the cervix is a rare entity. The most frequent of spread is a direct invasion as lymphatic and haematogenous spread is rare probably due to the relative small size of the cervix and the limited blood flow, the lymphatic drainage from the pelvis is away from the cervix and finally the fibrous tissue of the cervix. The ovaries 
The New Indian Journal of OBGYN. 2021 (July-December);8(1)

provide a more suitable environment for metastasis. The incidence being $25 \%$ compared to $3-8 \%$ in non-pregnant women with CRC. In a review by Mazur et al who reviewed 325 cases of metastases to the female genital tract he found only 12 cases were to the cervix, 143 cases to the ovary and 129 cases to the vagina ${ }^{8}$. In this case, we feel that there was a direct extension to the cervix and to the rectum that resulted in a rectovaginal fistula. Histopathology suggested there was no lymph vascular invasion.

Immunohistochemistry is useful in evaluating the origin of the primary site of the tumour cells. In our case a positive CK20 and CDX2 and a negative CK7 helped us to distinguish the existence of a colonic adenocarcinomas from other tumours. In addition the RAS oncogene wild types without mutation in exon 2 (codon 12,13) and 3 (codon 61) supports the underlying metastases.

Pregnant patients with CRC carry a poor prognosis. This is mainly due to a delayed diagnosis and lack of standardized guideline for treatment. Poor prognostic factors such as, a poorly differentiated and mucinous histology, advanced disease with perforation or obstruction, metastases, and incomplete resection of the tumors have been implicated in the survival rate.

The treatment options for CRC in pregnancy should be no different than the non-pregnant population. CT and MRI can be used in pregnancy after a discussion with the patient in terms of the risk and benefits. Proctosigmoidoscopy is helpful in the diagnosis as most cases of CRC in pregnancy are rectal cancers and a review by Bernstein et al showed the approximately $64-86 \%$ of $\mathrm{CRC}$ lies below the peritoneal reflection ${ }^{9}$.

\section{Conclusion}

Colorectal cancer is a rare entity and carries a poor prognosis as it usually presents in an advanced stage as in this particular case. Gastrointestinal symptoms should not be overlooked and promptly evaluated both in pregnancy and in the postpartum period.

\section{Conflict of interest: None. Disclaimer: Nil. References}

1. Girad RG, Lamarche J, Baillot R. Carninoma of the colon associated with pregnancy: report of a case. Diseases of the Colon and Rectum. 1981; 24(6): 473-5.
2. Saif MW. Management of colorectal cancer in pregnancy: a multimodality approach. Clin Colorectal Cancer. 2005; 5(4): 247-56.

3. Pellino G, Simillis C, Kontovounisios C, Baird DL, Nikolaou S, Warren $\mathrm{O}$, et al. Colorectal cancer diagnosed during pregnancy: systematic review and treatment pathways. Eur J Gastroenterol Hepatol. 2017; 29(7):743-53.

4. Slattery ML, Samowitz WS, Holden JA. Estrogen and Progesterone receptors in colon tumours. American Journal of Clinical Pathology. 2000; 113(3): 364-8.

5. Singh S, Shepard MC, Langman MJS. Sex differences in the incidence of colorectal cancer: an exploration of oestrogen and progesterone receptors. Gut. 1993; 34(5): 611-5.

6. Silverstein J, Kidder W, Fisher S, Hope TA, Maisel S, Dianna $\mathrm{Ng}$, et al, Hormone receptor expression of colorectal cancer diagnosed during the peri-partum period, Endocrine connections. 2019; 8(8): 1149-58.

7. The Cancer Genome Atlas Network. Comprehensive molecular characterization of human colon and rectal cancer, Nature. 2012; 487(7407): 330-7.

8. Mazur MT, Hsueh S, Gersell DG. Metastases to the female genital tract: analysis of 325 cases. Cancer. 1984; 53:1978-85.

9. Bernstein MA, Madoff RD, Caushaj PF. Colon and rectal in pregnancy. Dis Colon Rectum. 1993; 36:172-8.

\footnotetext{
Harjit S Dhaliwal ${ }^{1}$, Rachana Dwivedi ${ }^{2}$, Tharanga Gunasekera $^{3}$, Jasmina Glogic ${ }^{4}$

${ }^{1}$ Department of Obstetrics and Gynecology and Histopathology, Royal Bournemouth Hospital, Bournemouth, UK; ${ }^{2}$ Department of Obstetrics and Gynecology and Histopathology, Royal Bournemouth Hospital, Bournemouth, UK; ${ }^{3}$ Department of Obstetrics and Gynecology and Histopathology, Royal Bournemouth Hospital, Bournemouth, UK;

${ }^{4}$ Department of Obstetrics and Gynecology and Histopathology, Royal Bournemouth Hospital, Bournemouth, UK.
} 